\title{
Commenting on photoreactions of anthracene derivatives in the solid state
}

\author{
Tommaso Salzillo ${ }^{1,2}$ and Aldo Brillante ${ }^{1}$ \\ ${ }^{1}$ Dipartimento di Chimica Industriale “Toso Montanari”, Università di Bologna and INSTM-UdR \\ Bologna, Viale Risorgimento 4, 40136 Bologna, Italy. \\ ${ }^{2}$ Department of Molecular Nanoscience and Organic Materials, Institut de Ciència de Materials de \\ Barcelona (ICMAB-CSIC) and (CIBER-BBN), Campus de la UAB, 08193 Bellaterra, Spain.
}

\begin{abstract}
We review a series of photochemical reactions related to the renowned family of anthracene derivatives. We first classify the photodimerizations of 9-methyl-anthracene, 9-cyano-anthracene and 9-anthracene-carboxylic acid as topochemical, non-topochemical and reversible, respectively. We then introduce the so called topophysical reactions as the case when the lattice change involves two different molecular species. In the latter case the compatibility of the reactive species occurs at the level of a symmetry matching of the unit cells rather than going via a correct registry of molecular orientations. We then describe the relationship between incoming photons and mechanical motion, a common occurrence in crystal-to-crystal photoreactions, which reaches stunning effects when 9,10dinitro-anthracene is transformed to anthraquinone. Finally the kinetics of these systems is shortly discussed. By means of a micro-Raman technique we are capable of monitoring, at the same time and on the very same spot, the molecular (intramolecular vibrations or internal motions) and the lattice (intermolecular vibrations or lattice phonons) transformations. It turns out that molecular changes start first, producing, in a second time, the symmetry modifications of the crystal lattice. The extent of the delay of the structural change may considerably vary depending upon the system studied.
\end{abstract}

\footnotetext{
*Correspondence to:

aldo.brillante@unibo.it

tommaso.salzillo@unibo.it; tsalzillo@icmab.es
} 


\section{Introduction.}

When photons interact with the periodic lattice of a molecular crystal, excitons are formed as quasi-particles which propagate throughout the ordered structure like waves, carrying momentum hk. Historical descriptions of molecular excitations in solids can be found in the literature ${ }^{1-5}$.

The process following the exciton formation leads to a number of phenomena producing either collective radiative and non-radiative decays of the excited molecules or chemical and physical changes inside the unit cell. Crystal-to-crystals photoreactions belong to the latter category of nonradiative processes, following light absorption. Examples of this kind are numerous in the literature and the mechanisms underlying this class of experiments have been widely discussed in recent, as well as in the past, years ${ }^{6-10}$.

Photoreactions in the solid state are basically different from their analogous in solution, in that, in the former, the excitation propagates in the ordered structure of the crystal to eventually be selectively localized in the active sites of the reactant lattice that trigger the transformation to the product. In the attempt to describe the kinetic evolution of a photoreaction it is worth following the representation of a cage as the place where all chemical and physical changes occur ${ }^{11}$. In the crystal the cage is the unit cell, and its equivalent in solution is the perfect fit of dimerized molecules in a confined environment ${ }^{12}$

An interesting aspect of most photoreactions in the solid state is that, since the product itself is crystalline, they can be classified as crystal-to-crystal reactions. It is therefore convenient to describe the reaction in the solid state as a combination of two processes:

1) the photo-chemical change of the molecules inside the cage (intra-molecular process) and

2) the photo-physical transformation of the unit cell in the new lattice, that is, in a new environment of dispersive energy (inter-molecular process).

It is well documented ${ }^{13-15}$ that the two processes are not concomitant, the molecular change coming first, followed, with a diversified time delay, by the physical transformation of the lattice.

It is also worth to outline that in the initial stage of the reaction a number of product molecules, with a variable concentration, reside as guests in the host lattice of the reactant. Quite remarkably, cases have been described where this amount of product in the reactant lattice can persist up to an amazing $85 \%{ }^{16}$. Besides, the route to products may not necessarily be a crystalline state without lack of continuity, the amorphous form being a possible final state of the new species ${ }^{16}$. In this paper we comment on the outcome of crystal-to-crystal photoreactions by a qualitative classification of the variety of transformations occurring in different molecular crystals, all limited to the sample system of anthracene derivatives. 


\section{The Experiment.}

The approach to the study of crystal-to-crystal photoreactions and their evolution in time is naturally multidisciplinary, although, among the various techniques that can be employed to study the problem, X-ray diffraction (XRD) has been traditionally the technique of choice.

For the studies here described, we have followed the reaction by means of Raman microscopy, a consolidated technique which easily probes, in a single scan, the evolution in time of both the chemical transformation, by checking the variation of the internal modes, and the crystal structure modification. The latter is performed in the low wavenumber region $\left(10-150 \mathrm{~cm}^{-1}\right)$, where lattice phonons are active ${ }^{13}$. Lattice phonons are, in fact, collective translational and rotational motions of the molecules inside the unit cell and therefore are very sensitive to even slight packing or conformational changes of the crystal. This is of great importance, since permits to simultaneously follow the evolution of the reaction of the two processes, whose time scale, is not the same.

A further notable advantage of the micro-spectroscopy is the use of confocality, which enables to study the reaction in situ and with a spatial resolution below $1 \mu \mathrm{m}$, thus with the possibility to discriminate reacted and unreacted domains in the same specimen, up to the point to draw Raman maps ${ }^{17}$. Furthermore, by changing the numerical aperture (NA) of the microscope objective, different depths of the crystal can be probed, gathering information on whether the reaction is only confined to the surface or propagates into the bulk ${ }^{18}$.

The Raman setup used for all the experiments related to the examples shown in the paper consists of a triple monochromator from Horiba-Jobin Yvon coupled to an optical microscope Olympus BX40. A set of microscope objectives allowed us to obtain a maximum spatial resolution just below $900 \mathrm{~nm}$ and a nominal field depth ranging from about 7 to $25 \mu \mathrm{m}$. The excitation wavelength was from a krypton laser tuned at $647.1 \mathrm{~nm}$, an energy sufficiently low to avoid both background fluorescence from the sample as well as any competitive photochemical process. The incoming power was reduced with a neutral filter whose optical density was varied in each experiment to prevent crystal damage, the actual power focused on the sample being anyway less than $1 \mathrm{~mW}$. Lattice phonon spectral scans were run in the region $10-150 \mathrm{~cm}^{-1}$, whereas intramolecular vibrations were detected up to $1800 \mathrm{~cm}^{-1}$.

The irradiation procedures of the photo-excitation of the single crystals, broadband and/or laser, are described in detail in the referenced papers. A complete report on the Raman experiment can be found elsewhere ${ }^{19}$.

\section{Crystal-to-Crystal Photochemical Reactions.}


We classify and compare the possible cases that can be encountered when a reaction driven by light leads to new products in the crystalline state.

1) Topochemical.

This is the case where the energy transfer and the chemical transformation itself are favored by the mutual molecular orientations and positions in the crystal structure of the reactant molecules, that implies their transformation with a minimum positional reconstruction. This is the so called topochemical principle, dating back to the early work of the group of Schmidt ${ }^{6,20-23}$ and subsequently applied to a diacetylene polimerization ${ }^{24-26}$. Its central idea states that the product molecules are geometrically preformed in the crystal packing of the original lattice. A survey of the early experiments on the subject can be found in the literature ${ }^{9,27}$. A most recent example is documented for 9-methylanthracene (9MA) ${ }^{15,28}$.

Figure 1a (structures as inset) shows how the monomer molecules of 9MA in the unit cell constitute a perfect fit for their dimerization to the trans dimer (9MAD). Indeed, after only few minutes of standard irradiation the reaction proceeds very rapidly to its full completion.
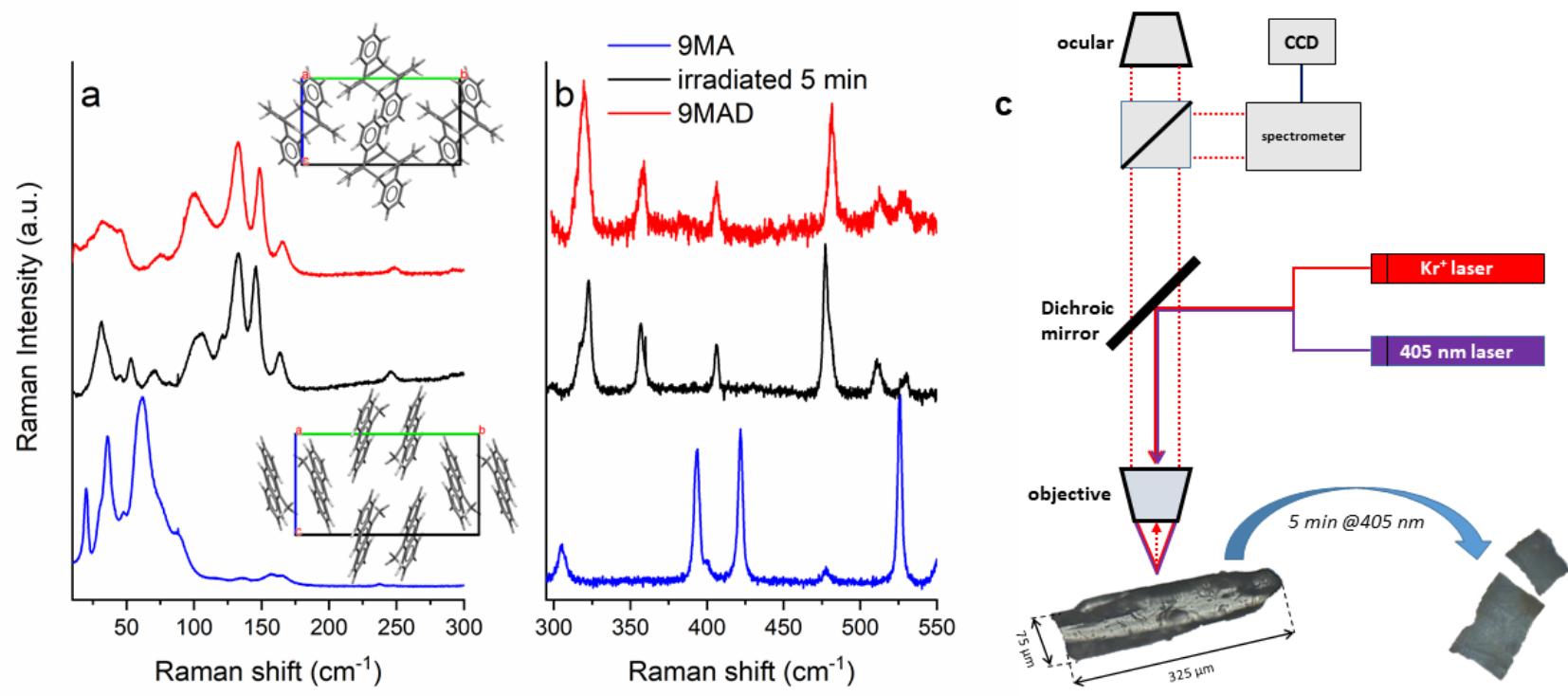

Figure 1 Structure of the monomer and the dimer of 9MA (insets) and spectral evolution of the reaction in the lattice phonon region (a) and in the 300-600 $\mathrm{cm}^{-1}$ intra-molecular region (b). A sketch of the experiment is also reported (c).

Raman spectra neatly show the complete molecular transformation of the monomer to the dimer as indicated by the internal vibrations above $200 \mathrm{~cm}^{-1}$ (Figure 1b). On the contrary the corresponding changes of the lattice phonons modes directly monitor that also the transformation of the unit cell has occurred (Figure 1a). The reaction has then reached its full completion, both from the chemical and the physical point of view, which agrees with the expectation that a topochemical 
mechanism would imply the process to progress fast and with a high yield after irradiation. In other words we believe that reaction rate and reaction yield are two non-separable factors in solid state reactions, both driving the outcome of the reaction.

Lastly, the figure also shows that the crystal breaks in uneven bits after irradiation (bottom part of Figure 1c). Fragmentation of the crystal is a recurrent outcome of this as well of most photoreactions described in the paper, irrespective of the incoming irradiation power used. It is intrinsically related to the physico-chemical change and its explanation lies on the energetics of the process: reacted and unreacted crystal domains during the photoreaction produce strain at the boundaries, resulting in movements and breaking of the sample.

2) Non-topochemical.

In this case there is no positional correlation among reactant and product molecules in the unit cell and the outcome of the photoreaction cannot be foreseen by the only inspection of the initial crystal structure. Noticeable example is the historical case of 9-cyano-anthracene (9CNA) originally reported by Craig and Sarti Fantoni ${ }^{29}$ and more recently revisited ${ }^{14}$. Here the molecules are arranged in the lattice in such a way to give a head-to-head, or cis, fashion but the topochemical formation of the dimer (9CNAD) is inhibited due to the steric hindrance arising from having both substituents on the same side. What is observed instead is the formation of the trans or head-to-tail dimer ${ }^{29-31}$. Figure 2 shows the spectral evolution of the photodimerization reaction. The surprising result is explained by assuming that the reaction starts in defective sites of the crystal lying on dislocations where crystal planes are shifted in a way that pairs of molecules are in a head-to-tail registry. This assumption was later confirmed by XRD experiments ${ }^{30}$, that showed how along the (221) planes the molecules were indeed pre-arranged as pairs in the trans configuration. The idea of the advancing front of reacted molecules acting as further defect sites, conveys the hint of an autocatalytic process which permits the reaction to spread further. This is the reason why the mechanism of non-topochemical photoreactions is often referred to as driven by defects ${ }^{6,29,31-33}$. 


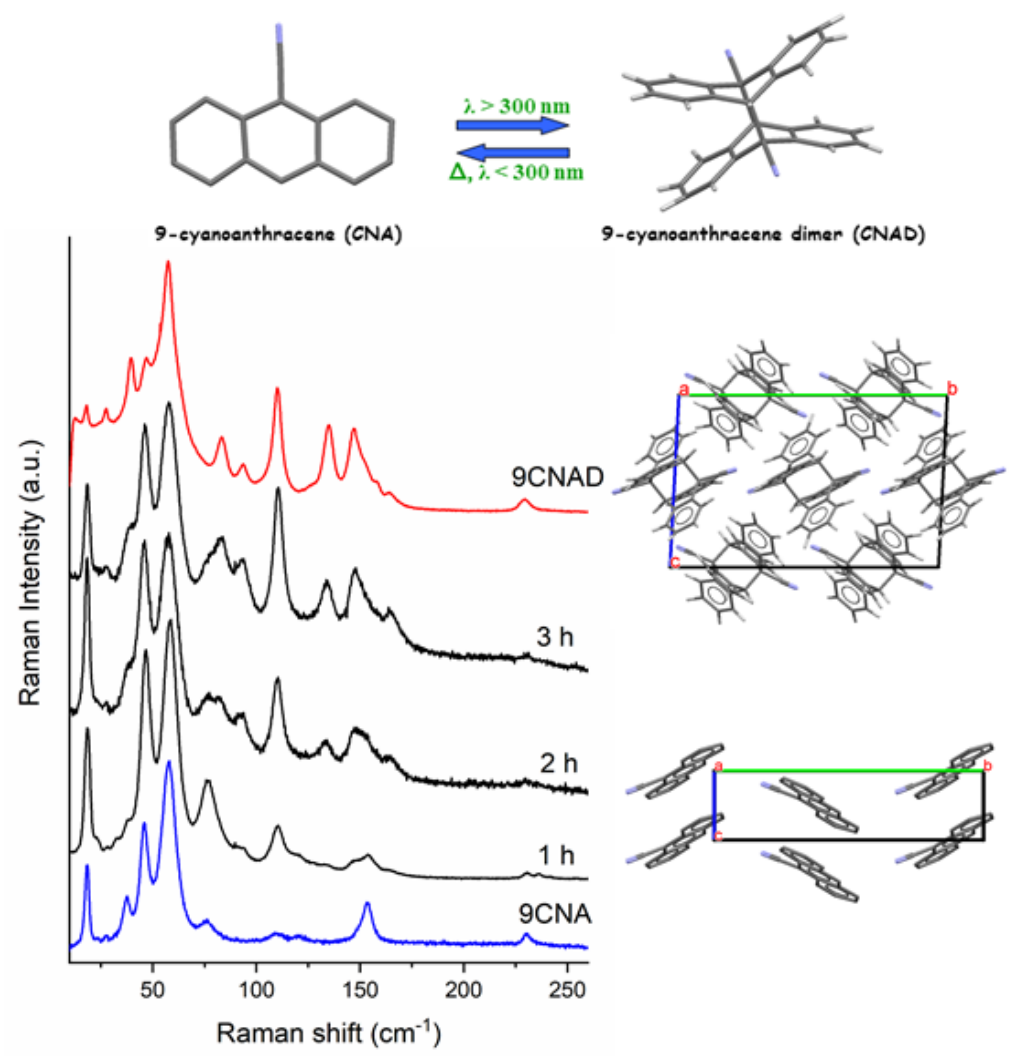

Figure 2 Photodimerization scheme of 9CNA (upper part) and spectral evolution of the reaction in the lattice phonon region (lower part). Structures of the monomer and of the photodimer are drawn aside their corresponding spectra.

It is interesting to comment on the mechanism at the base of these two classes of photodimerization reactions. In both cases, the topochemical and the non-topochemical one, the preceding step of the photochemical change is the presence of a local pseudo-dimeric state given by a configuration of pairs of molecules in a face-to-face fashion. This state has been described as a kind of physical dimer, that, following exciton theory, has been subsequently called an excimer state ${ }^{34}$. The excimer (or excited dimer) is a localized state consisting of two coupled molecules sharing the excitation after light irradiation ${ }^{4,34}$. The fate of the excimer depends on the different efficiency of the competing radiative and non-radiative decay channels following excitation. When the former prevails, we observe the characteristic broad and red shifted photoluminescence typical of the excimer emission. On the contrary, in the latter case, a radiationless process occurs, leading to the chemical union between the two identical molecules, whose bonds did not originally exist. In this view the excimer is recognized as the precursor of the chemical photodimer ${ }^{35}$, which convincingly explains the topochemical mechanism. The process is qualitatively sketched in Figure 3. 


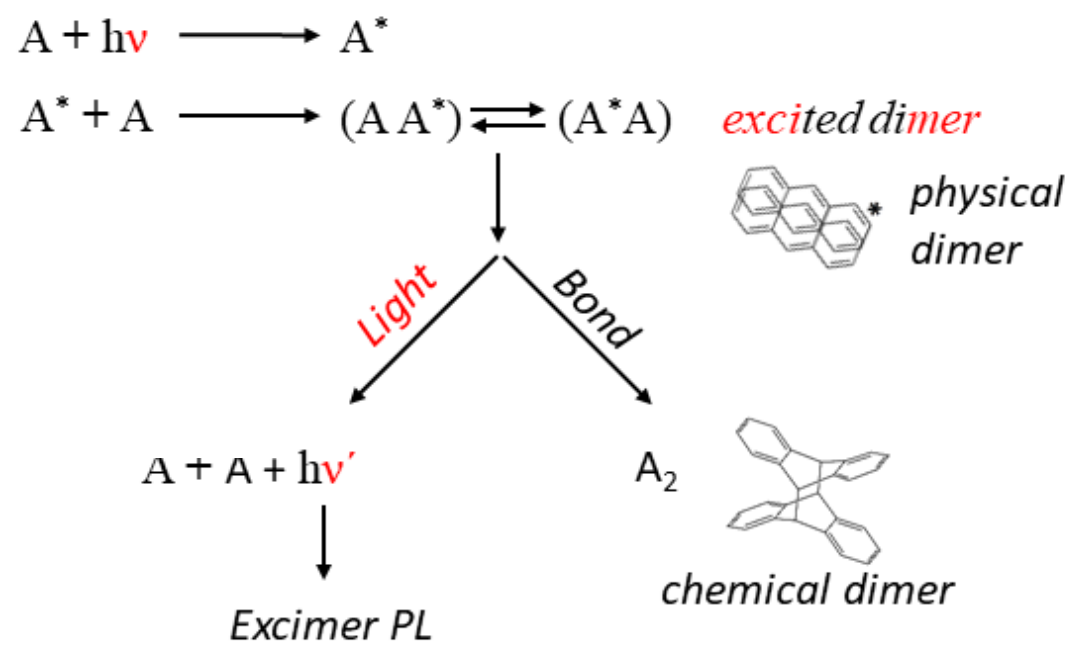

Figure 3 Radiative (left) and non-radiative (right) decay of the excimer state. The excimer (dimeric) photoluminescence (PL) occurs at a frequency $v<<v$, the exciton (monomeric) PL.

The photodimerization of anthracene itself is an intriguing case which falls in this category. Its occurrence in solution dates back to $1866^{36}$, but what is surprising is its dimerization in the solid state. In fact, unlike the stacked structure of many of its derivatives, the arrangement of the molecules in the crystal is herringbone-like $\mathrm{e}^{37,38}$, a situation where apparently there is no way to have a face-toface correct registry for the [4+4] topochemical cyclo-addition. Nevertheless, under UV irradiation, it is easy to reach the formation of its dimer ${ }^{39}$, via a mechanism driven by defects similar to that previously described for 9CNA. In this case the defective zones are mainly located on the crystal surface, showing dislocations of crystal planes which produce domains of pairs of molecules oriented face-to-face, that is, correctly arranged for dimer formation ${ }^{40}$. Also in this case the photoreaction obviously proceeds in an autocatalytic way.

Figure 4 shows the evolution of the initial lattice phonon spectrum of anthracene (AN, blue trace) to that of the dimer (AND), drawn as reference in the red trace. It takes a number of hours of broadband uv irradiation with a $75 \mathrm{~W}$ xenon lamp to begin to observe the spectral features of the AND. The enlarged picture of the irradiated crystal (picture on the top of the the spectral profile in the figure) shows that large domains of dimerized molecules appear as white stripes, on the surface of the specimen, which constitute the reactive front of the photodimerization. 

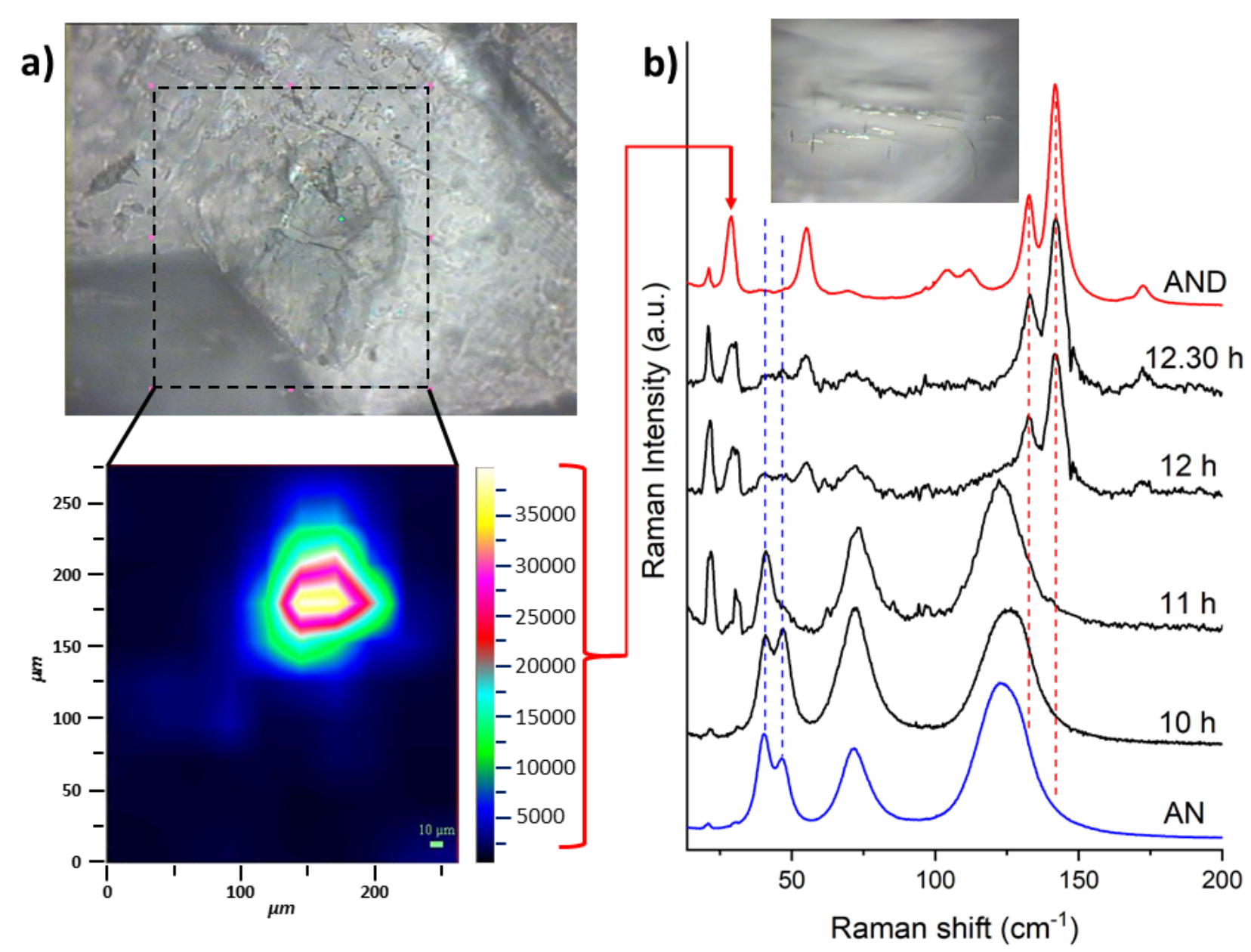

Figure 4 Right side: Spectral evolution of lattice phonon spectra from the monomer (blue trace) to the dimer (black traces). The reference spectra of the dimer is in red. In the inset an enlarged view of dimerized domains. Left side: Raman map (lower part) of the photodimerization for a specimen shown in the upper part. The dotted vertical lines mark the intense spectral features of AN (disappearing) and AND (appearing) during the photoreaction. For details see text.

In order to show how the reaction is triggered and subsequently propagates, we show in the left part of the figure a Raman $\operatorname{map}^{17}$ of a portion of a monomer specimen about 250x250 micrometers, as indicated. The amount of the dimer formation has been obtained by monitoring the intensity of the phonon peak at about $30 \mathrm{~cm}^{-1}$, a spectral window where no signal of the monomer is observed. The increase of the dimer intensity is represented with a palette of false colors, which visually indicates the point of the crystal where the photoreaction has started (white spot) and the extent of its propagation.

Recently the anthracene photodimerization was re-investigated controlling the reaction atmosphere ${ }^{41}$. In ambient atmosphere the reaction occurs as reported by previous works, whereas by using an oxygen enriched atmosphere it proceeds by an intermediate stage with the formation of the 9,10-anthracene-endoperoxide ${ }^{42}$, which only in a second stage decomposes to anthraquinone.

3) Reversible. 
It may happen that a chemical transformation begins, but does not proceed up to its full completion, actually at some point returning to the starting point. This is the case of 9anthracenecarboxylic acid (9ACA), a molecule where the photoreaction takes place very quickly in solution $^{43}$, where in a number of solvents the head-to-tail photodimer is easily obtained ${ }^{44}$. Interestingly, the single crystals of the unsolvated 9ACA dimer (9ACAD) could be obtained only by precipitation from acetone. For all other solvents used, only solvate structures were found, with a variable stoichiometric ratio of dimer and solvent molecules. A complete account of all solvates of 9ACAD identified, and whose structures have been solved by X-ray diffraction, has been recently given $^{44}$.

Once the reaction is replicated in the solid, a partial formation of photodimers is observed ${ }^{44}$ ${ }^{47}$, whose common feature is that, when the light is switched off, the system reverts to its original chemical and physical state.

It has been recently reported that 9ACA shows polymorphism, crystallizing in two different systems: monoclinic (9ACA- $\alpha$ ) and triclinic (9ACA- $\beta$ ), both with two molecules per unit cell. It was found that in many solution-grown batches the two polymorphs are concomitant, whereas by using xylenes or ethyl acetate as solvents it was possible to obtain the pure $\alpha$ and $\beta$ phase, respectively ${ }^{46}$. In our experiments we have tested selectively single crystals of both forms to check a possible different reactivity under irradiation.

Following the procedures previously described, we have used Raman microscopy to produce this short-lived dimer, and showed that we needed a couple of hours of standard irradiation to weaken the spectral features of the monomer in its triclinic form (9ACA- $\alpha$ ) at the expenses of a new series of bands assigned to the concomitant head-to-head metastable photodimer ${ }^{42}$ (Figure $5 \mathrm{a}$ ). The pictures in the inset show a partial rotation of the crystal, reminiscent of the twisting of the microribbons as observed a few years ago $^{28}$. However, further irradiation did not produce any advancement of the reaction. On the contrary, once the crystal was left in the dark, the full phonon pattern of the monomer was restored.

We thus classify this photoreaction as reversible. 

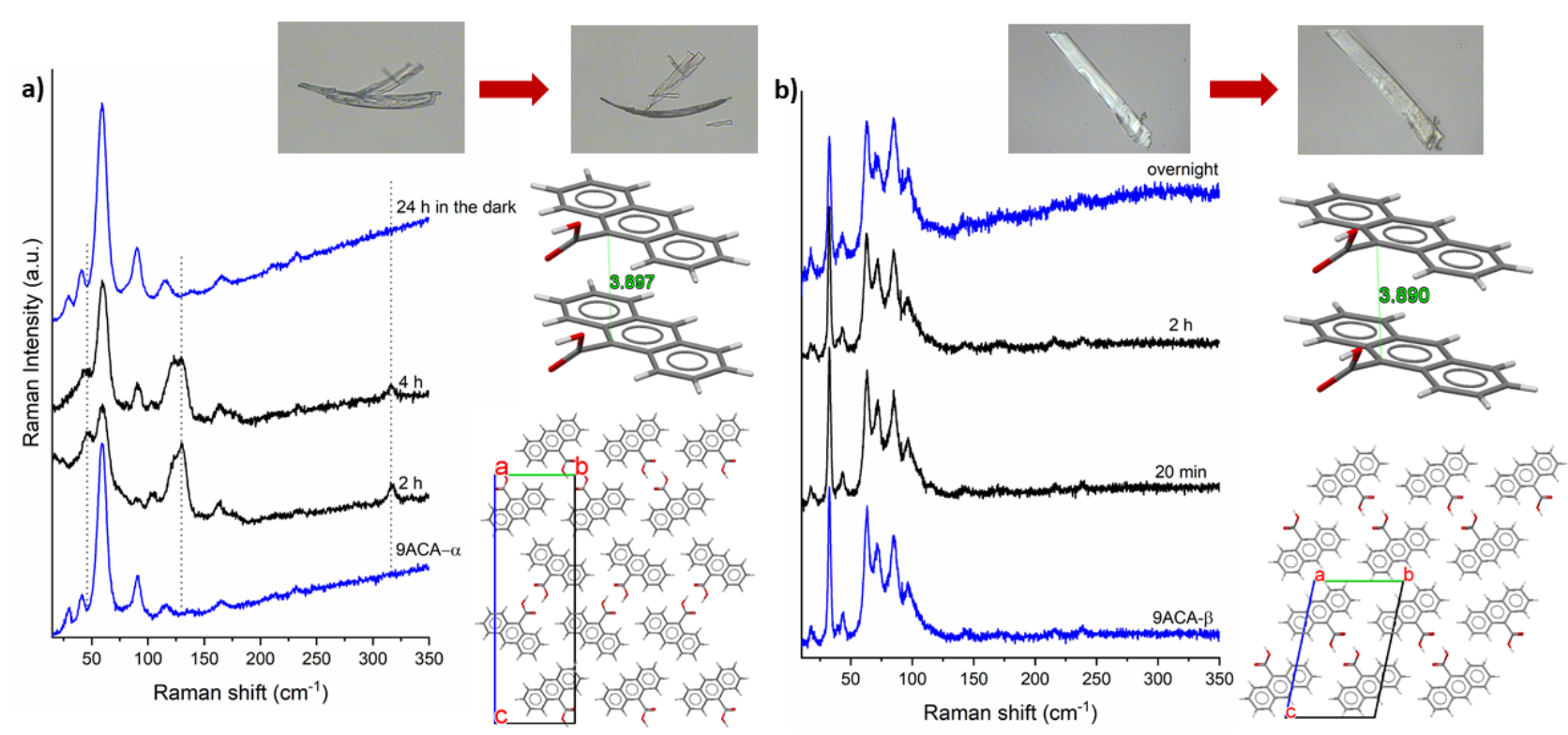

Figure 5 Lattice phonon Raman spectra and crystal structures of the monoclinic (a) and of the triclinic (b) 9ACA crystals irradiated by an unfocused diode laser at $405 \mathrm{~nm}$. In the inset the images of the irradiated specimens are shown.

Once the same procedure is performed on crystals of the $\beta$ phase (Figure $5 b$ ), in all cases we apparently did not observe any modification of the lattice phonon spectra, even after prolonged overnight irradiation, although the picture of the crystal (inset of figure 5b) shows that somehow the sample had undergone some morphological change. The initial bright surface of the single crystal turns to be opaque after the irradiation, with no more homogeneous extinction at the polarizing microscope. It may well be that the different packing of the $\beta$ polymorph prevents any reactivity along the 9ACA stacks. The suggestive hypothesis of a reversible reaction even faster than that observed for the $\alpha$ polymorph could be anyway considered.

By inspecting the crystal structures, reported in figure 5, we observe that both polymorphs make columnar stacks of their molecular planes, with very similar distances between intra-columnar anthracene cores, 3.90 and $3.89 \AA$ for the $\alpha$ and $\beta$, respectively, thus both favorable to the formation of the cis-photodimer, according to the topochemical principle. However, the $\mathrm{COOH}$ groups are rotated with respect to the anthracene cores, making hydrogen bonds between neighboring molecules of the stacks. The main difference between the two polymorphs is the orientation of the different paired columnar stacks. While for the $\alpha$ form the pairs are rotated by $62.7^{\circ}$ in the $\beta$ form they are perfectly parallel to each other. While it has been reported that it is doubtful that these differences can affect the photoreactivity of the monomer ${ }^{44,45}$, it has been also reported that different ratio of the concomitant $\alpha$ and $\beta$ forms in nanorods and nanoribbons can generate a different photoresponse ${ }^{48}$. In other words it is tempting to think that these different orientations of the stacks can in some way affect the instability of the photodimers in the host lattice, for instance making even faster the 
monomerization of the process for the polymorph $\beta$ with larger steric hindrance of molecular pairs in the stack.

4) Topophysical.

This can be described as the case where the perfect fit which drives the reaction to the photoproduct is not given by the organization of the molecules in the crystal, but, in a large extent, by the mutual compatibility of reactant and product at the level of symmetry of the unit cell. In this sense this particular photochemical process can be described as topophysical.

The exemplary reaction here reported is the transformation of 9,10-dinitroanthracene $\left(\mathrm{DNO}_{2} \mathrm{~A}\right)$ to anthraquinone $(\mathrm{AQ})+2 \mathrm{NO}^{13}$. Since we are not dealing here with a photodimerization, the mechanism is not bound to the initial formation of the excimer state nor to its non-radiative decay to a chemical dimer. The reaction is instead strongly driven by the space group symmetry of the species into play. We start with a triclinic cell with a number of molecules $Z=1\left(\mathrm{DNO}_{2} \mathrm{~A}\right)$ to end up with a monoclinic cell with $\mathrm{Z}=2$ (AQ) and about double the volume, with a sizable contraction of about $14 \%$ due to the loss of $2 \mathrm{NO}$. By looking at the structural parameters of $\mathrm{DNO}_{2} \mathrm{~A}$ and $\mathrm{AQ}$ unit cells (table 1$)^{49-52}$, we do notice that a reaction mechanism can be drawn on the basis of a cell doubling along a suitable direction, leading to an efficient physical fit between the two lattices. This might justify why the reaction goes so easily to completion. The reaction path has, in fact, been successfully modeled and a full account of the reaction can be found in the literature ${ }^{13}$.

Table 1 Cell parameters for $\mathrm{DNO}_{2} \mathrm{~A}$ and $\mathrm{AQ}$

\begin{tabular}{r|rr}
$\mathbf{I D}$ & $\mathbf{D N O}_{2} \mathbf{A}^{49}$ & $\mathbf{A Q}^{51}$ \\
\hline Space group & $\mathrm{P}-1$ & $\mathrm{P}_{1} / \mathrm{c} 11$ \\
$\mathbf{Z}$ & 1 & 2 \\
$\mathbf{V}\left(\mathbf{\AA}^{3}\right)$ & 278.586 & 478.602 \\
$\boldsymbol{a}(\AA)$ & $3.95(1)$ & $3.956(3)$ \\
$\boldsymbol{b}(\AA)$ & $8.68(2)$ & $7.866(3)$ \\
$\mathbf{c}(\AA)$ & $8.76(2)$ & $15.766(4)$ \\
$\boldsymbol{\alpha}\left(^{(}\right)$ & $106.77(17)$ & $102.7(3)$ \\
$\boldsymbol{\beta}\left(^{\circ}\right)$ & $98.98(17)$ & 90 \\
$\gamma\left(\left(^{\circ}\right)\right.$ & $98.02(17)$ & 90
\end{tabular}

In figure 6 we report a couple of schematic simulation of cell-doubling which can occur during the photoprocess. This explains why the reaction goes so easily to completion. The reaction path has also been successfully modelled and a full account of the reaction can be found in the literature ${ }^{13}$. 

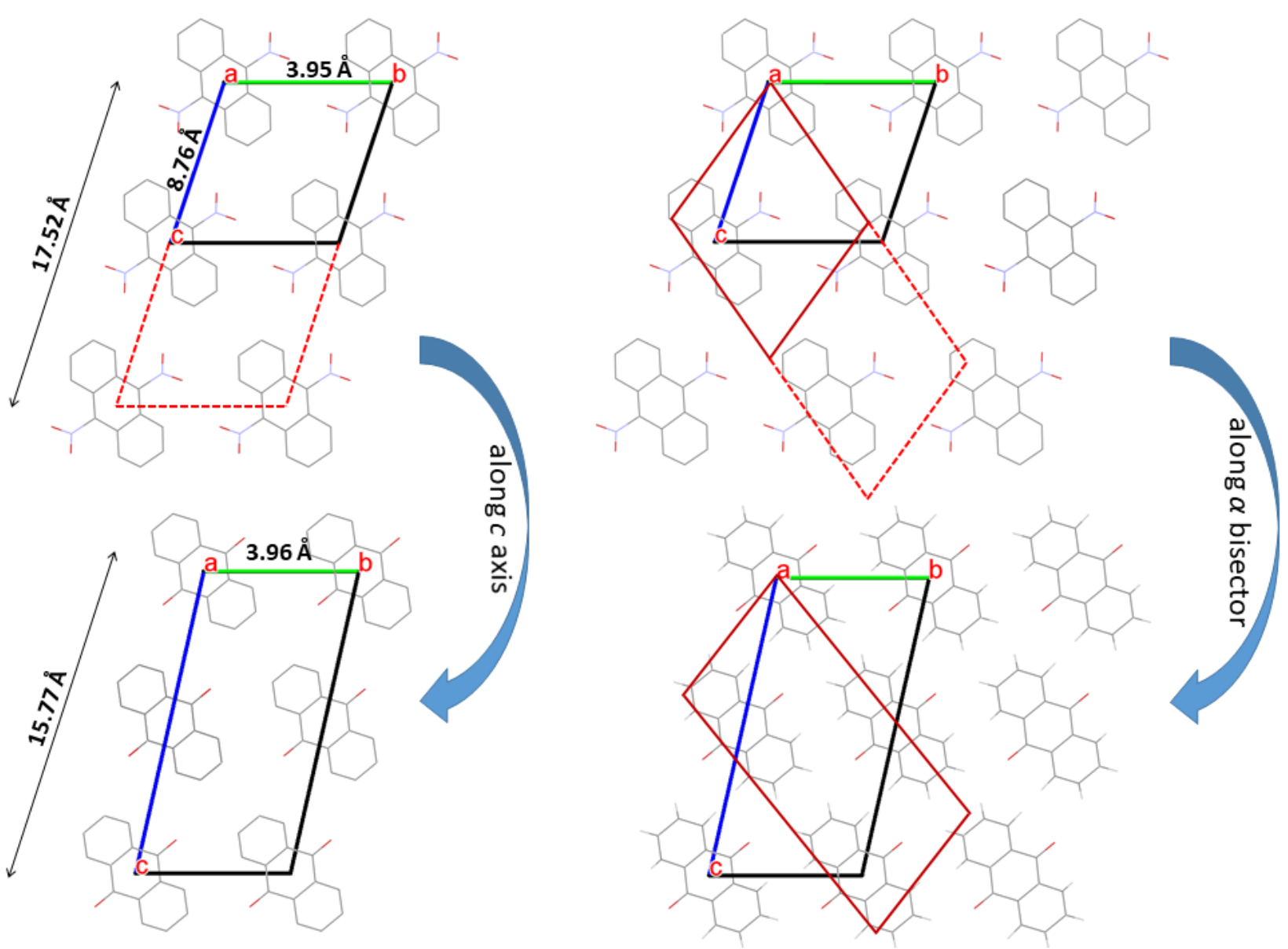

Figure 6 graphical scheme of hypothesis of cell-doubling along c axis (left) and a projection along alpha angle bisector (right).

The left part of figure 7 shows the evolution of the reaction as monitored by confocal microRaman spectroscopy in the lattice phonon region: the phonon spectrum of $\mathrm{DNO}_{2} \mathrm{~A}$ fully converts to the corresponding spectrum of pure anthraquinone ${ }^{13}$. This outcome is accompanied by the observation of a spectacular series of snapshots reported in the right part of the figure, where a striking series of mechanical motions, bending, twisting and unfolding, accompanies the transformation of the single crystal of $\mathrm{DNO}_{2} \mathrm{~A}$ into AQ. An interesting observation needs to be focused at this stage. The wellshaped initial single crystal, apparently recovers its initial shape after $365 \mathrm{~min}$, though in a non isooriented form, but the spectral profile indicates that residual bands of $\mathrm{DNO}_{2} \mathrm{~A}$ are still present. The completion of the photoreaction requires that the residual $\mathrm{DNO}_{2} \mathrm{~A}$ molecules react and a corresponding stoichiometric amount of NO molecules leaves the crystal, which further shrinks to keep its cohesion. At this stage only, the reaction ends. 

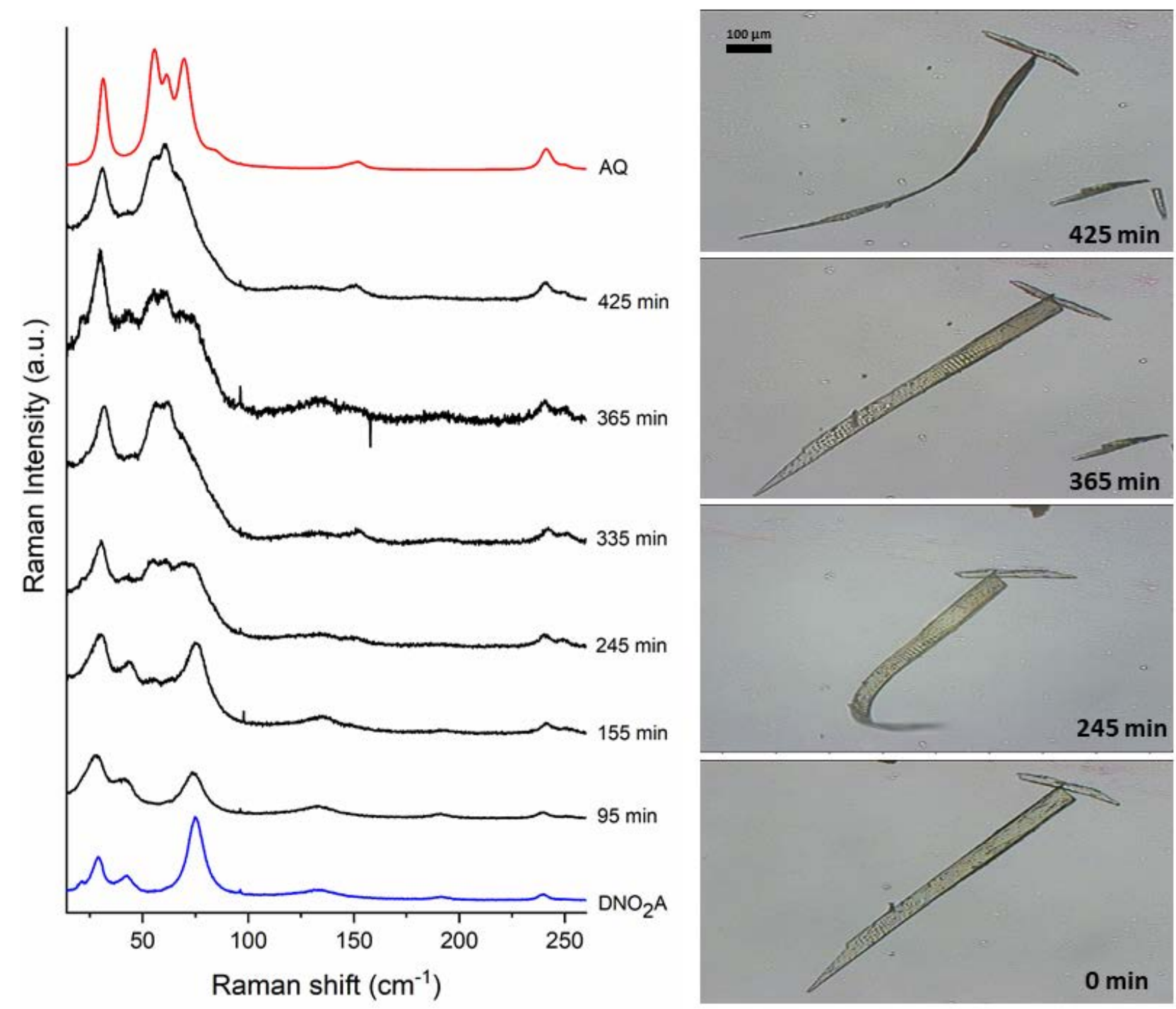

Figure 7 Lattice phonon Raman spectra of a DNO2A single crystal as a function of time under uniform irradiation from a defocused UV laser beam and selected snapshots, at the corresponding times, of the irradiated crystal during the course of the reaction.

Similar trends have been observed for other crystals of approximately the same shape and size. A Windows media video file documenting this spectacular behaviour is available as Supplementary Information of ref ${ }^{13}$.

\section{Photons and mechanical motion}

The mechanical response following light absorption can be exploited both at supramolecular level $^{53-55}$ or for the entire crystal ${ }^{13,56-66}$. While in the former case the effort was finalized in pursuing the achievement of molecular machines, the increasing interest of the latter is linked to the search for mechanically responsive crystals as systems with a high potential for the conversion of radiative energy into mechanical work (photomechanical actuators). 
It is a common feature of the crystal-to-crystal photoreactions that, even in conditions where the topochemical principle holds, a large structural reconstruction is involved during the photoreaction. The consequence on the original crystal is a strong mechanical stress, driven by the incoming photons, which ends up to change the macroscopic shape of the crystal with a series of movements up to the breaking, or even to the explosion, of the specimen. Comprehensive descriptions of these findings are summarized in refs ${ }^{67,68}$.

The conversion of the photon energy into mechanical motion is certainly due to the relaxation of the strain produced in the crystal as long as new guest (product) molecules take the place on the very same sites of the host (reactant) ones. These are called substitutional positions of the original lattice. Phenomena of this kind are similar to those encountered when the contribution to the lattice reconstruction is given by temperature or pressure.

With reference to the anthracenes derivatives here described, regardless to which category the photoreaction belongs, mechanical motion is always accompanying the chemical and physical changes occurring during their transformation. The behavior ranges from the stunning acrobatics of photoirradiated $\mathrm{DNO}_{2} \mathrm{~A}^{13}$, to the fragmentation of single crystals of $9 \mathrm{MA}^{15,28}$ and to the reversible motions of microribbons of 9ACA ${ }^{46-48}$. Therefore, even in those cases, classified as topochemical, where the lattice reconstruction is minimal, the reactant monomers still undergo significant mechanical response to the light stimulus. However, it is worth to notice that, as observed in 9MA, by attenuating the intensity of the incoming light, the fragmentation of the single crystals may be drastically reduced ${ }^{15}$, enabling to conveniently follow the photodimerization of the single crystals up to its completion.

Slight crystal movements have also been reported for non topochemical photodimerizations ${ }^{14}$. In these cases, since the reactions start in defective regions of the crystal, the strain produced by the concomitant presence of domains of reacted and unreacted molecules is comparable to that of the topochemical reactions.

Finally, for the reversible photodimerization of 9ACA, also the mechanical response of nanorods and nanoribbons is described as reversible ${ }^{46-48,58}$, hinting interesting perspectives for technological applications.

\section{The Reaction Kinetics.}

It is of great importance to understand the mechanism underlying the crystal-to-crystal transformation, a process complicated by the need to consider all changes occurring in the molecular environment during the reaction. As already mentioned, the interesting point of the photoreactions in the solid state is that the process to follow is twofold, the one related to the molecular transformation, 
i.e. changes inside the unit cell, and the second concerning the physical change, i.e., the symmetry transformation collectively involving the crystal as a whole. Since the former obviously comes first, the question is how much delay will be detected between the two processes and how different, in terms of rate of reaction, would it be. Indeed, the extent of the delay may considerably vary, and energetics (structure) and dynamics (lattice phonons) of the crystal will both be involved. The delay in time between the two events is certainly dependent, for a given system, on sample history and size of the starting material. In any case two distinct kinetic laws need always to be identified. This is certainly the most interesting phenomenon which is revealed by our experimental approach to the problem. While the reaction proceeds, former guest molecules (the early content of photoproduct) eventually become the host molecules, while the former hosts (the molecules of the reactant) take the place of the guests. This picture, spreading from the cage, the unit cell, to the whole crystal, represents the key point to explain the reaction mechanism in the solid state in all its stages. Investigations at this purpose are not easy, since it would require complex kinetics experiments for every specific reaction into play. However, since the spectroscopic technique can monitor, in a single experiment and at the very same times, both the molecular and the unit cell changes, it is necessary to collect a series of spectral scans at given times. By playing with the intensity changes of the Raman bands, the weakening of those of the monomer crystal at the expenses of the growing intensity of the dimer bands, one can try to draw their dependence in time, in order to rationalize their outcome in terms of a kinetic law.

The example here described refers to the photodimerization of 9CNA. Figure 8 shows the conversion factor of reactant molecules, $\alpha$, as a function of time, with emphasis to the initial onset of the reaction, the one triggered by defects. Whereas the blue line represents the chemical transformation (from intramolecular modes) the red one is related to the physical transformation (from lattice phonons). The full kinetic treatment is extensively described in $\operatorname{ref}^{14}$. 


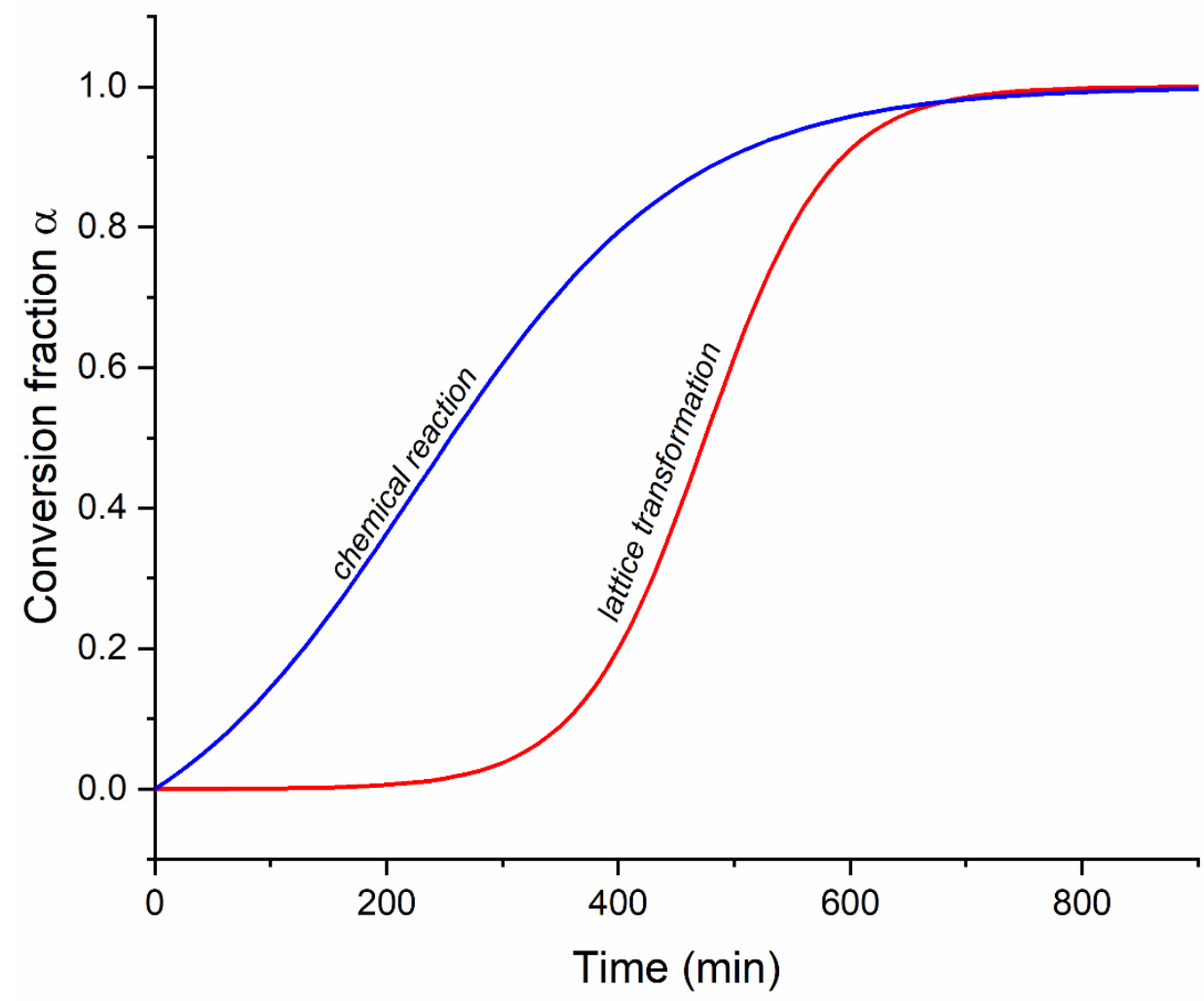

Figure 8 Conversion fraction $\alpha$ as a function of time as obtained from the band intensities of micro-Raman spectra. Lines: fitted curves according to the FW eq 1. of ref 14.

The observed time mismatch between chemical and lattice transformation is self evident by looking at the figure. At a time when more that $50 \%$ of molecules are transformed, the change of the spatial group symmetry has not yet started. Though qualitatively expected, the extent of this delay at first sight might look really surprising. This order of magnitude was already observed for the completely different topophysical case of $\mathrm{DNO}_{2} \mathrm{~A}$ transformed to $\mathrm{AQ}$. An accurate spectroscopic estimate of the transformation of reactant to product molecules, yielded a value of $40 \%$ of photoproduct (AQ) occupying substitutional sites of the unchanged original triclinic lattice of the reactant $\left(\mathrm{DNO}_{2} \mathrm{~A}\right)$, as discussed and modeled in Ref ${ }^{13}$. A following paper from Garibay group ${ }^{16}$ has gone even beyond these numbers. An amazing amount of up to 85\% of guest molecules in the host lattice of the reactant was detected by XRD measurements of crystalline triazolines transformed quantitatively by denitrogenation to aziridines in a solid-to-solid reaction.

Therefore, the paradigm that substitutions of the molecules in the crystal lattice cannot survive at high concentration of the guest ${ }^{69}$ needs to be reconsidered. We believe that this point deserves more attention in consideration of these unpredictable amounts of transformed molecules still in the host lattice and focused experiments are worth to be planned at the purpose.

In conclusion, although it is clear that molecular changes start first to produce following modifications of the crystal lattice, depending on the system studied, the extent of the delay of the 
structural change may considerably vary following the different routes underlying each class of photoreactions, leading to different kinetics as well as to different yields.

\section{Conclusions}

We have described a series of photochemical reactions in the solid state, all belonging to the class of anthracenes. Despite we are dealing with the same class of compounds, we found a variable phenomenology that required a simplified classification of their outcome. Four distinct cases have been encountered: topochemical, non-topochemical, reversible and topophysical. Though by no mean we reckon this classification is complete, we believe that it may be anyway useful to separate the mechanisms underlying each class of these photoinduced reactions.

We have discussed the role of the two species, reactant (host) and product (guest), in the crystal, finding intriguing situations on the amount of substitutional guest molecules in the host lattice. In the cases described the product itself has always be found crystalline, and in this respect they can be classified as crystal-to-crystal reactions. The initial single crystal ends up in most case with a mosaic-like collection of microcrystal, obviously no longer iso-oriented. In practically all cases, the change is accompanied by macroscopic mechanical deformations, spanning explosion, fragmentation or sort of acrobatic movements. The strain at the borders of reacted and unreacted domains is certainly the reason of this occurrence, though no clear signature of the lattice deformation could be seen in the Raman spectra, nor, at our knowledge, could be found in the XRD experiments reported in the literature.

Once more, we should emphasize the successful use of the micro-Raman technique in monitoring the reaction at the chemical and physical level of the transformation. The method is fast, versatile, non destructive and in situ with spatial resolution below 1 micrometer. Despite all these advantages, however, we still believe that XRD support is a must to complementary accompany the spectroscopic results.

Additional results are reported on the kinetics of these transformations. Regardless of the kind of crystal-to-crystal transformation, we systematically identified a different time delay between the molecular change, which comes first, and the following change of the lattice into the unit cell of the product. In one case (9CNA) this occurrence was carefully quantitatively analyzed, separating the intra-molecular changes from the inter-molecular ones. One cannot certainly neglect the history of each sample and its chemical and physical purity, as a relevant factor of a different yield or of a different kinetics. The role of defects certainly needs to be further examined. It is not a paradox that a perfect crystal would never react, since the excitons produced by the incoming light will be completely de-localized and hence not capable of producing any decay. 
Finally we outline that the structural modifications are always strongly mediated by the lattice phonons which couple to the electronic excitation. Structure and dynamics of the crystal are then equally involved in a crystal-to-crystal photoreaction and this should be taken into account when designing every new reaction.

\section{REFERENCES}

$1 \quad$ J. Frenkel, Phys. Rev., 1931, 37, 1276-1294.

$2 \quad$ J. Frenkel, Phys. Rev., 1931, 37, 17-44.

3 A. S. Davydov, Theory of molecular excitons, McGraw Hill, New York, 1962.

4 D. P. Craig and S. H. Walmsley, Excitons in molecular crystals, theory and applications, New York, 1968.

5 S. Nakajima, Y. Toyozawa and R. Abe, The Physics of Elementary Excitations, SpringerVerlag Berlin Heidelberg, Springer S., 1980.

6 M. D. Cohen, in Reactivity of solids, eds. J. S. Anderson, M. W. Roberts and F. S. Stone, Springer US, 1st edn., 1972, pp. 456-471.

7 A. E. Keating and M. A. Garcia-Garibay, in Organic and Inorganic Photochemistry, eds. V. Ramamurthy and K. Schanze, Marcel Dekker, New York, 1998, p. 195.

8 K. Tanaka and F. Toda, in Organic solid state reactions, ed. F. Toda, Springer, Dordrech, 2002, pp. 109-158.

9 M. Kaftory, in Supramolecular photochemistry: controlling photochemical processes., eds. V. Ramamurthy and Y. Inoue, John Wiley \& Sons, Hoboken, New York, 2011, pp. 229-266.

10 Y. Ohashi, Crystalline State Photoreactions, Springer Japan, Tokyo, 2014.

11 D. P. Craig, R. Soc. New South Wales, 1982, 115, 61-67.

12 M. Yoshizawa, Y. Takeyama, T. Okano and M. Fujita, J. Am. Chem. Soc., 2003, 125, 32433247.

13 T. Salzillo, I. Bilotti, R. G. Della Valle, E. Venuti and A. Brillante, J. Am. Chem. Soc., , DOI:10.1021/ja307088n.

14 T. Salzillo, S. Zaccheroni, R. G. Della Valle, E. Venuti and A. Brillante, J. Phys. Chem. C, 2014, 118, 9628-9635.

15 T. Salzillo, E. Venuti, R. G. Della Valle and A. Brillante, J. Raman Spectrosc., 2017, 48, 271-277.

16 D. De Loera, A. Stopin and M. A. Garcia-Garibay, J. Am. Chem. Soc., 2013, 135, 66266632.

17 A. Brillante, I. Bilotti, R. G. Della Valle, E. Venuti, M. Masino and A. Girlando, Adv. Mater., 2005, 17, 2549-2553.

18 A. Rivalta, T. Salzillo, E. Venuti, R. G. Della Valle, B. Sokolovič, O. Werzer and A. Brillante, ACS Omega, 2018, 3, 9564-9571.

19 A. Brillante, I. Bilotti, R. G. Della Valle, E. Venuti and A. Girlando, CrystEngComm, 2008, 10, 937-946.

20 M. D. Cohen and G. M. Schmidt, J. Chem. Soc., 1964, 1996-2000.

21 S. T. P. I. I. I and B. G. M. J. Schmidt, J. Chem. Soc., 1964, 2014-2021.

22 G. M. J. Schmidt, Pure Appl. Chem., 1971, 27, 647-678.

23 G. M. J. Schmidt, Solid state photochemistry, Verlag Chemie, Weinheim, Germany, 1976.

24 G. Wegner, Z. Naturforschg, 1969, 24b, 824-832.

25 G. Wegner, Die Makromol. Chemie, 1972, 154, 35-48.

26 V. Enkelmann, in Polydiacetylenes. Advances in Polymer Science, ed. H. J. Cantow, Springer, Berlin, Heidelberg, 1984. 
V. Ramamurthy and K. Venkatesan, Chem. Rev., 1987, 87, 433-481.

28 T. Kim, L. Zhu, L. J. Mueller and C. J. Bardeen, J. Am. Chem. Soc., 2014, 136, 6617-6625.

29 D. P. Craig and P. Sarti-Fantoni, Chem. Commun., 1966, 20, 742-743.

30 M. D. Cohen, Z. Ludmer, J. M. Thomas and J. O. Williams, Proc. R. Soc. Lond. A, 1971, 324, 459-468.

31 Z. Ludmer, Chem. Phys., 1977, 26, 113-121.

32 M. D. Cohen, Tetrahedron, 1987, 43, 1211-1224.

33 A. Brillante, M. Cau, R. G. Della Valle and E. Venuti, Chem. Phys. Lett., 1994, 218, 568573.

34 M. Pope and C. E. Swenberg, Electronic Processes in Organic Crystals and Polymers, Oxford UNiversity Press, New York, 2nd editio., 1999.

35 V. Ramamurthy and Y. Inoue, Eds., Supramolecular photochemistry: controlling photochemical processes., John Wiley \& Sons, Hoboken, New York, 2011.

36 J. Fritzsche, J. Prakt. Chem., 1867, 101, 333.

37 A. M. Mathieson, J. M. Robertson and V. C. Sinclair, Acta Crystallogr., 1950, 2, 245-250.

38 C. P. Brock and J. D. Dunitz, Acta Crystallogr. Sect. B, 1990, 46, 795-806.

39 A. P. Rood, D. Emerson and H. J. Milledge, Proc. R. Soc. London. A. Math. Phys. Sci., 1971, 324, 37-43.

40 T. Seko, K. Ogura, Y. Kawakami, H. Sugino, H. Toyotama and J. Tanaka, Chem. Phys. Lett., 1998, 291, 438-444.

41 M. Lee, C. Park, J. Kim, J. Lee, S. Kim, J. Y. Koo and H. C. Choi, ACS Appl. Mater. Interfaces, 2018, 10, 33773-33778.

42 A. Lauer, A. L. Dobryakov, S. A. Kovalenko, H. Fidder and K. Heyne, Phys. Chem. Chem. Phys., 2011, 13, 8723-8732.

43 T. Wolff and N. Müller, J. Photochem., 1983, 23, 131-140.

44 T. Salzillo, E. Venuti, C. Femoni, R. G. Della Valle, R. Tarroni and A. Brillante, Cryst. Growth Des., 2017, 17, 3361-3370.

45 R. Morè, G. Busse, J. Hallmann, C. Paulmann, M. Scholz and S. Techert, J. Phys. Chem. C, 2010, 114, 4142-4148.

46 L. Zhu, R. O. Al-Kaysi, R. J. Dillon, F. S. Tham and C. J. Bardeen, Cryst. Growth Des., 2011, 11, 4975-4983.

47 L. Zhu, R. O. Al-Kaysi and C. J. Bardeen, J. Am. Chem. Soc., 2011, 133, 12569-12575.

48 J. T. Good, J. J. Burdett and C. J. Bardeen, Small, 2009, 5, 2902-2909.

49 J. Trotter, Acta Crystallogr., 1959, 12, 232-236.

50 K. Lonsdale, H. J. Milledge and K. El Sayed, Acta Crystallogr., 1966, 20, 1-13.

51 A. T. H. Lenstra and J. F. J. Van Loock, Bull. Soc. Chim. Belg., 1984, 93, 1053-1055.

52 Y. Fu and C. P. Brock, Acta Crystallogr. Sect. B Struct. Sci., 1998, 54, 308-315.

53 V. Balzani, A. Credi, F. Raymo and J. F. Stoddart, Artificial Molecular Machines., 2000, vol. 39.

54 V. Balzani, A. Credi and M. Venturi, Molecular Devices and Machines - A Journey into the Nano World., Wiley-VCH, Weinheim, Germany, 2003.

55 M. Von Delius and D. A. Leigh, Chem. Soc. Rev., 2011, 40, 3656-3676.

56 M. Irie, Science (80-. )., 2001, 291, 1769-1772.

57 C. Weder, J. Mater. Chem., 2011, 21, 8235.

58 T. Kim, M. K. Al-Muhanna, S. D. Al-Suwaidan, R. O. Al-Kaysi and C. J. Bardeen, Angew. Chemie - Int. Ed., 2013, 52, 6889-6893.

59 R. O. Al-Kaysi, A. M. Müller and C. J. Bardeen, J. Am. Chem. Soc., 2006, 128, 1593815939.

60 M. A. Garcia-Garibay, Angew. Chemie - Int. Ed., 2007, 46, 8945-8947.

61 E. R. Kay, D. A. Leigh and F. Zerbetto, Synthetic molecular motors and mechanical machines, 2007, vol. 46. 
62 S. Kobatake, S. Takami, H. Muto, T. Ishikawa and M. Irie, Nature, 2007, 446, 778-781.

63 K. Uchida, S. I. Sukata, Y. Matsuzawa, M. Akazawa, J. J. D. De Jong, N. Katsonis, Y.

Kojima, S. Nakamura, J. Areephong, A. Meetsma and B. L. Feringa, Chem. Commun., 2008, 326-328.

64 H. Koshima, N. Ojima and H. Uchimoto, J. Am. Chem. Soc., 2009, 131, 6890-6891.

65 M. Morimoto and M. Irie, J. Am. Chem. Soc., 2010, 132, 14172-14178.

66 H. Koshima, K. Takechi, H. Uchimoto, M. Shiro and D. Hashizume, Chem. Commun., 2011, 47, 11423-11425.

67 N. K. Nath, M. K. Panda, S. C. Sahoo and P. Naumov, CrystEngComm, 2014, 16, 18501858.

68 T. Kim, L. Zhu, R. O. Al-Kaysi and C. J. Bardeen, ChemPhysChem, 2014, 15, 400-414.

69 A. Brillante and D. P. Craig, J. Chem. Soc. Faraday Trans. 2 Mol. Chem. Phys., 1975, 71, 1457-1472. 